\title{
EFECTO DE TRES DILUTORES EN LA CONSERVACIÓN DEL SEMEN DE ALPACAS
}

\author{
Fernando Raymundo T. ${ }^{1}$, Wilfredo Huanca L. ${ }^{2}$, Teodosio Huanca M. ${ }^{3}$, \\ Sandra Huerta O. ${ }^{1}$ y Aída Cordero R. ${ }^{4}$
}

\section{Abstract}

The present work was carried out at the Experimental Research Station QuimsachataINIA, Puno. The objective was to evaluate the efficiency of three semen extenders in alpaca semen: Tris-glucose, Tris-fructose and a pig's commercial extender. Twelve animals were selected for semen collection using the artificial vagina. Males were trained for a month. Mean values for semen parameters were: volume of $2.7 \pm 0.8 \mathrm{ml}$, viscosity of 1.04 \pm 0.3 , motility of $54.0 \pm 8.0 \%, \mathrm{pH}$ towards to alkaline, concentration of $248,000 \mathrm{sperms} / \mathrm{ml}$, and the most common color was milky white. The average time for the copula was $26.5 \pm$ 3.8 minutes. Semen was diluted in 1:2 and the dilutions were evaluated on individual motility as the only parameter for sperm viability. The extender Tris-glucose had an average of $5.8 \pm 1.1$ hours viability, Tris-fructose had $6.1 \pm 2.5$ hours, and the commercial extender had $5.5 \pm 1.0$ hours, without statistical differences between extenders.

Key words: extender, semen, alpaca

\section{Resumen}

El presente trabajo tuvo el propósito de evaluar la eficiencia de tres dilutores: Trisglucosa, Tris-fructosa y un dilutor comercial de cerdo, en la conservación del semen de alpaca. Se utilizaron 12 machos que fueron entrenados por un mes en la colección de semen con vagina artificial y frazadilla eléctrica. Los animales fueron de la Sub-Estación Experimental Quimsachata del INIA, Puno. El semen tuvo las siguientes características: volumen de $2.7 \pm 0.8 \mathrm{ml}$, viscosidad de $1.04 \pm 0.3$, motilidad de $54.0 \pm 8.0 \%$, pH con tendencia a la alcalinidad, concentración de 248,100 espermatozoides $/ \mathrm{ml}$, y el color que predominó fue el blanco lechoso. El tiempo promedio de cópula fue de $26.5 \pm 3.8$ minutos. Se utilizó un factor de dilución de 1 en 2 para semen y dilutor, respectivamente. Las diluciones fueron evaluadas considerando la motilidad individual como único parámetro para determinar la viabilidad espermática. El dilutor Tris-glucosa mostró una viabilidad promedio de $5.8 \pm 1.1$ horas, el Tris-fructosa de $6.1 \pm 2.5$ horas y el dilutor comercial de cerdo de $5.5 \pm 1.0$ horas, sin haber diferencia estadística significativa entre dilutores.

Palabras clave: dilutor, semen, alpaca

\author{
${ }^{1}$ Práctica privada \\ ${ }^{2}$ Laboratorio de Reproducción Animal, FMV-UNMSM. E-mail: wilfredo.huanca@gmail.com \\ ${ }^{3}$ Estación Experimental ILLPA-INIA, Puno \\ ${ }^{4}$ Facultad de Zootecnia, Universidad Nacional Agraria La Molina
}


INTRODUCCIÓN

La crianza de camélidos sudamericanos en las zonas alto-andinas del Perú es uno de los principales sustentos económicos de un amplio sector de la población rural, además de ser una fuente importante de proteína de origen animal y de abrigo; sin embargo, las condiciones de explotación de los camélidos sudamericanos en la mayor parte de las comunidades campesinas se realizan sin una adecuada atención técnica, ocasionando bajos índices reproductivos (Chipana, 1997).

El conocimiento de la biología reproductiva de los camélidos sudamericanos es aún insuficiente, especialmente en lo referido a la fisiología reproductiva del macho. La inseminación artificial es una de las tecnologías que ha contribuido al progreso genético en diversas especies de animales de producción, especialmente en el bovino; sin embargo, existe muy poca información sobre la colección, características, evaluación y conservación del semen en la alpaca, debido a la falta de una metodología confiable y reproducible para la colección de semen (Ferré y Werkmeister, 1996). Las principales limitaciones son la colección de semen, la falta de conocimiento sobre su composición, viscosidad y uso de dilutores (Bustinza, 2001).

El semen de la alpaca es altamente viscoso, lo que hace difícil su manejo en el laboratorio. Así mismo, la viscosidad del semen influye para que no exista la motilidad masal; además, la motilidad individual es de tipo oscilatoria y muy lenta (Sumar, 1997). Las características físicas del semen son muy semejantes a las de la llama (Garnica y Achata, 1998).

La dificultad en la colección del semen es atribuido entre otras causas, a las características peculiares de monta (posición de cúbito ventral), que dura entre 20 a 30 minutos, aproximadamente. Además los animales son de temperamento nervioso, lo que dificulta su manejo (Fernández-Baca y Calderón, 1965).
La conservación de las células espermáticas se basa, esencialmente, en el uso de dilutores que proporcionen los nutrientes adecuados, que neutralizen los cambios de $\mathrm{pH}$ producido por el metabolismo de los azúcares (efecto tampón), y que protejan a los espermatozoides del descenso de temperatura durante el proceso de congelación (Herrrera, 1986).

A la fecha, se reporta el uso de varios dilutores para semen de alpacas, entre los que se encuentran la leche descremada, fosfato salino tamponado, glucosa-citrato, yema de huevo-glucosa-citrato, tryladil y tris tamponado; aunque parece que el mejor dilutor para semen de alpacas es el tris tamponado (Bravo, 1989), existen resultados contradictorios y muchas veces no repetibles. El presente estudio tuvo como objetivo estudiar el efecto de tres dilutores en la conservación del semen de alpacas.

\section{Materiales y Métodos}

\section{Lugar de estudio}

El presente estudio se llevó a cabo durante los meses de febrero y marzo del 2000 en la sub-estación experimental Quimsachata, perteneciente al INIA (Instituto Nacional de Investigaciones Agrarias), ubicada en el distrito de Santa Lucía, provincia de Lampa, departamento de Puno. La zona tiene una altitud de 4,200 msnm y una temperatura de $27^{\circ} \mathrm{C}$ en los meses de junio a marzo. La precipitación pluvial promedio anual es de $688 \mathrm{~mm}$.

El análisis de los datos se realizó en el Laboratorio de Reproducción Animal de la Facultad de Medicina Veterinaria, Universidad Nacional Mayor de San Marcos.

\section{Selección y entrenamiento de los machos}

Se seleccionaron 12 animales de la raza Huacaya, cuyas edades fluctuaban entre 6 y 8 años, dentro de un total de 180 alpacas ma- 
Cuadro 1. Componentes de los dilutores de semen usados para la conservación de semen de alpacas

\begin{tabular}{lrlrl}
\hline \multicolumn{2}{c}{ Tris-Glucosa } & \multicolumn{2}{c}{ Tris-Fructosa } & Comercial de cerdo \\
\hline Ácido cítrico & $2.9 \mathrm{~g}$ & Ácido cítrico & $2.9 \mathrm{~g}$ & Glucosa \\
Fructosa & $1.25 \mathrm{~g}$ & Glucosa & $3 \mathrm{~g}$ & Citrato de sodio \\
Yema de huevo & $20 \%$ & Yema de huevo & $20 \%$ & EDTA \\
Penicilina & $10,000 \mathrm{UI}$ & Penicilina & $10,000 \mathrm{UI}$ & Cloruro de potasio \\
Estreptomicina & $100 \mathrm{mg}$ & Estreptomicina & $100 \mathrm{mg}$ & Lincomicina \\
& & & & Espectinomicina \\
\hline
\end{tabular}

chos. La selección se hizo básicamente considerando el interés del macho por el maniquí que tenía la forma de una hembra en celo. Además, se consideró la condición corporal, el estado sanitario, y el tamaño, forma, consistencia y elasticidad de los testículos y epidídimo, así como las condiciones del prepucio y el pene.

Los animales fueron entrenados 3 veces por semana durante 4 semanas antes del periodo experimental, para su acostumbramiento al personal, monta y uso de la vagina artificial. La temperatura interna de la vagina fue de $40{ }^{\circ} \mathrm{C}$. Se evaluaron las características seminales de motilidad, viscosidad, $\mathrm{pH}$, color y volumen. Se usó una frazadilla eléctrica para mantener constante la temperatura de la vagina artificial durante la monta.

\section{Dilutores}

Antes de la dilución, el semen fue aspirado con una jeringa equipada con una aguja fina de tuberculina, con la finalidad de reducir la viscosidad de manera mecánica. La dilución se hizo en una proporción de una parte de semen y dos de dilutor.
Se evaluaron los dilutores Tris-Glucosa, Tris-Fructosa y un dilutor comercial para semen de cerdos (Cuadro 1). La yema de huevo utilizada procedió de huevos recolectados el mismo día de la preparación del dilutor. Los dilutores se mantuvieron en baño maría a $37{ }^{\circ} \mathrm{C}$. En el caso del dilutor comercial de cerdo se siguieron las instrucciones del fabricante.

Las evaluaciones del semen diluido se hicieron con intervalos de una hora hasta encontrar una motilidad cero. La viabilidad espermática del semen diluido se evaluó considerando como única variable la motilidad individual, dado que la lectura del porcentaje de espermatozoides vivos y muertos no era confiable.

\section{Análisis estadístico}

Se determinó el promedio, desviación estándar, coeficiente de variación y valores extremos del volumen, motilidad, concentración espermática, viscosidad y $\mathrm{pH}$.

A través del análisis de tiempo a un evento (Curva de Kaplan Meier), se determinó el tiempo que tomó la motilidad 
espermática para llegar a cero, considerando el análisis de cada eyaculado en forma independiente.

\section{Resultados y Discusión}

Los $26.5 \pm 3.8$ minutos de cópula (rango de 12 a $47 \mathrm{~min}$.) registrado en el presente estudio (Cuadro 2) se encuentra en el rango reportado por otros autores. Así, FernándezBaca et al. (1970) encontró un tiempo de cópula de $22 \pm 1$ minuto en 44 servicios de monta controlada y Sucapuca (1991) de $27 \pm$ 9 minutos usando el método del preservativo.

El semen presentó un color variable entre cristalino a blanco lechoso; similar a lo reportado por Mogrovejo (1952) usando funda vaginal, Fernández-Baca (1964) con electroeyaculación, y Quispe y Olarte (1988) con vagina artificial y maniquí. La variabilidad de color puede ser atribuida a la concentración de espermatozoides en el eyaculado.

El aspecto del semen fue viscoso, similar a un gel, en todos los eyaculados. Esta característica se midió teniendo en cuenta la distancia de ruptura del semen cuando es levantado por una varilla (Cuadro 2), siguiendo la metodología reportada previamente (Fernández-Baca, 1964; Sumar y Leyva, 1981; Quispe y Olarte, 1988). El pH mostró una tendencia a la alcalinidad. El aspecto viscoso del semen es un factor que hace difícil la evaluación ya que no permite una correcta preparación de frotices (Fernández-Baca, 1964).

El eyaculado promedio tuvo un volumen de $2.7 \mathrm{ml}$ (Cuadro 2). Leyva et al. (1984) reportan volúmenes de $2.8 \pm 0.9 \mathrm{ml}$ y Dávalos et al. (1989) de $1.03 \pm 0.04 \mathrm{ml}$ en vagina artificial con maniquí y $1.73 \pm 0.09 \mathrm{ml}$ usando vagina artificial con hembra en celo. En general, los volúmenes obtenidos en los diferentes estudios difieren debido principalmente a la técnica utilizada (Bustinza, 2001).

La concentración espermática de la alpaca es muy variable, posiblemente debido a diferencias individuales y de método de colección (Sumar, 1997). En el presente estudio se encontró un valor de 248,100 espermatozoides por ml (Cuadro 2), similar al encontrado por Leyva et al. (1984) y Quispe (1987). Sin embargo, en publicaciones anteriores, se han reportado valores de 33,320 espermatozoides/ml (Mogrovejo, 1952) usando funda vaginal y de 48,000 espermatozoides/ml (Fernández Baca y Calderón, 1965) con electroeyaculación.

La motilidad fue muy variable, fluctuando entre el 30 al $90 \%$. Esta característica depende de la variación individual y del método de colección. Así, Mogrovejo (1952) usando funda vaginal encontró una motilidad

Cuadro 2. Características del eyaculado de 12 alpacas utilizando vagina artificial y maniquí de hembra

\begin{tabular}{lccc}
\hline & Promedio \pm d.e. & Mínimo & Máximo \\
\hline Cópula (minutos) & $26.5 \pm 3.8$ & 12 & 47 \\
Viscosidad (cm) & $1.04 \pm 0.28$ & 0.1 & 2.5 \\
Volumen (ml) & $2.7 \pm 0.8$ & 0.2 & 5.0 \\
Concentración (esperm./ml) & 248,100 & 96,000 & 474,000 \\
Motilidad $(\%)$ & $54.0 \pm 8.0$ & 30 & 90 \\
\hline
\end{tabular}


Cuadro 3. Tiempo promedio de supervivencia espermática (motilidad) en semen de alpacas utilizando tres tipos de dilutores de semen

\begin{tabular}{lccc}
\hline Dilutor & $\begin{array}{c}\text { Promedio } \\
\text { (horas) }\end{array}$ & $\begin{array}{c}\text { Máximo } \\
\text { (horas) }\end{array}$ & $\begin{array}{c}\text { Mínimo } \\
\text { (horas) }\end{array}$ \\
\hline Tris-Glucosa & $5.8 \pm 1.1$ & 10 & 4 \\
Tris-Fructosa & $6.1 \pm 2.5$ & 10 & 4 \\
Comercial de cerdo & $5.5 \pm 1.0$ & 11 & 3 \\
\hline
\end{tabular}

de 20-40\%, Fernández Baca y Calderón (1965) con electroeyaculación encontró entre 50 al $60 \%$ y Bravo (1989) usando vagina artificial encontró una motilidad de $30 \%$.

No se obtuvo ventaja significativa para ninguno de los tres dilutores en evaluación. Además, la duración de la motilidad fue pobre (Cuadro 3).

\section{Conclusiones}

- No se obtuvieron diferencias estadísticas significativas en la conservación del semen de alpacas a $36^{\circ} \mathrm{C}$ entre los dilutores TRIS-glucosa, TRIS-fructosa y un dilutor comercial de cerdo.

- Las características seminales fueron: volumen: $2.7 \pm 0.8 \mathrm{ml}$; concentración: $248,100 \pm 16,000$ espermatozoides $/ \mathrm{ml}$; motilidad: $54 \pm 8 \%$; viscosidad: $1.035 \pm$ 0.28 ; color blanco lechoso y $\mathrm{pH}$ ligeramente alcalino.

\section{Literatura Citada}

1. Bravo, F. 1989. Estudio comparativo de tres métodos de colección de semen en alpacas. Tesis de Bachiller. Facultad de
Zootecnia, Univ. Nacional Agraria La Molina. Lima. 96 p.

2. Bustinza, V. 2001. La alpaca. p 158184. Oficina de Recursos de Aprendizaje. Perú.

3. Chipana, Q.O. 1997. Colección de semen y evaluación de dilutores sobre la viabilidad espermática de alpacas. Tesis de Bachiller. Facultad de Zootecnia, Univ. Nacional Agraria La Molina. Lima. 78 p.

4. Dávalos, R.; J. Olazábal; L. Echevarría. 1989. Avances en la evaluación de dos formas de colección de semen en alpacas. Resúmenes II Congreso Mundial sobre Camélidos. Cuzco. p 74.

5. Fernández Baca, S. 1964. Algunas consideraciones sobre los métodos de colección de semen de la alpaca. Anales II Congreso Nacional de Veterinaria y Zootecnia. Lima. p 188-190.

6. Fernández Baca, S.; W. Calderón. 1965. Métodos de colección de semen de alpaca. Bol. IVITA. Lima. 12 p.

7. Fernández Baca, S.; D. Madden; C. Novoa. 1970. Effect of different mating stimuli on induction of ovulation in the alpaca. J. Reprod. Fertil. 22: 261-267.

8. Ferré, L.; A. Werkmeister. 1996. Desarrollo de una vagina artificial termoeléctrica para la colecta de semen. Rev. Agr. Prod. Anim. 16: 363-365.

9. Garnica, J.; R. Achata. 1998. Constituyentes químicos del plasma seminal de la alpaca. Resúmenes XII Reunión. Asoc. Per. Prod. Anim. Lima. p 66. 
10. Herrera, E. 1986. Evaluación de dilutores para la conservación de semen en ovinos. Tesis de Bachiller. Facultad de Medicina Veterinaria y Zootecnia, Univ. Nacional del Altiplano. Puno. 72 p.

11. Leyva, V.; J. Sumar; E. Franco. 1984. Estudio preliminar de la concentración de espermatozoides de semen de alpaca obtenida con vagina artificial. Resúmenes III Reunión Asoc. Per. Prod. Anim. Lima. p 18-21.

12. Mogrovejo, D. 1952. Estudio del semen de la alpaca. Tesis de Bachiller. Facultad de Medicina Veterinaria, Univ. Nacional Mayor de San Marcos. Lima. 27 p.

13. Quispe, F. 1987. Evaluación de las características físicas del semen de alpaca durante la época de empadre. Tesis de Bachiller. Facultad de Medicina Veterinaria y Zootecnia, Univ. Nacional del Altiplano. Puno. 70 p.
14. Quispe, F.; U. Olarte. 1988. Comparativo sexual y colección de semen de la alpaca en el periodo de un año. VI Conv. Internac. de Especialistas en Camélidos. Bolivia.

15. Sucapuca, V. 1991. Características físicas del semen de la alpaca obtenida por el método del preservativo. Tesis de Bachiller. Facultad de Medicina Veterinaria y Zootecnia, Univ. Nacional del Altiplano. Puno. $58 \mathrm{p}$.

16. Sumar, J.; V. Leyva. 1981. Colección de semen mediante vagina artificial en la alpaca (Lama pacos). Resúmenes IV Conv. Internac. sobre Camélidos Sudamericanos. Chile. p 3-4.

17. Sumar, J. 1997. Avances y perspectivas en reproducción de camélidos. I Symposium Internacional Avances en Reproducción de Rumiantes. Lima. p 30. 